\title{
3D-cultured adipose tissue-derived stem cells inhibit liver cancer cell migration and invasion through suppressing epithelial-mesenchymal transition
}

\author{
HAIHUA XIE ${ }^{1 *}$, NAISHUN LIAO ${ }^{2,3 *}$, FENGHUA LAN $^{1}$, ZHIXIONG CAI $^{2,3}$, \\ XIAOLONG LIU ${ }^{2,3}$ and JINGFENG LIU ${ }^{2-4}$ \\ ${ }^{1}$ Department of Clinical Genetics and Experimental Medicine, Fuzong Clinical College, Fujian Medical University; \\ ${ }^{2}$ The United Innovation of Mengchao Hepatobiliary Technology Key Laboratory of Fujian Province, \\ Mengchao Hepatobiliary Hospital of Fujian Medical University; ${ }^{3}$ The Liver Center of Fujian Province, \\ Fujian Medical University, Fuzhou, Fujian 350025; ${ }^{4}$ Liver Disease Center, The First Affiliated Hospital \\ of Fujian Medical University, Fuzhou, Fujian 350007, P.R. China
}

Received July 5, 2017; Accepted December 13, 2017

DOI: $10.3892 /$ ijmm.2017.3336

\begin{abstract}
Adipose tissue-derived stem cells (ADSCs) are considered promising candidates for stem cell therapy; however, the tumorigenicity of ADSCs remains controversial. The present study aimed to investigate the association between ADSCs and liver cancer cells, and to determine whether culture methods could influence the effects of ADSCs on liver cancer cell growth in vitro. Liver cancer cells were treated with ADSCs-conditioned medium (CM) that was collected using the two-dimensional (2D) culture method, sphere culture method, or three-dimensional (3D) culture method. After that, cell viability and apoptosis were measured using CCK-8 and Annexin V-FITC assay, respectively; the cell motility and adhesive capacity were analyzed by scratch wound healing and cell adhesion assay, respectively; the cell migration and invasion were examined by Transwell units; and the molecular mechanisms of ADSCs on effecting epithelial mesenchymal transition signaling pathway were further analyzed. The results demonstrated that ADSCs-CM was able to inhibit the growth of liver cancer cells by inhibiting cell proliferation and promoting cell apoptosis, as well as by suppressing cell motility, adhesive capacity, migration and invasion. In addition, ADSCs-CM was able to suppress cell growth via the downregulation of epithelial-mesenchymal transition signaling. Notably,
\end{abstract}

Correspondence to: Professor Xiaolong Liu or Professor Jingfeng Liu, The United Innovation of Mengchao Hepatobiliary Technology Key Laboratory of Fujian Province, Mengchao Hepatobiliary Hospital of Fujian Medical University, 312 Xihong Road, Fuzhou, Fujian 350025, P.R. China

E-mail: xiaoloong.liu@gmail.com

E-mail: drjingfeng@126.com

*Contributed equally

Key words: adipose tissue-derived stem cells, liver cancer cells, migration, invasion, epithelial-mesenchymal transition the enhanced inhibitory effects of ADSCs on liver cancer cell growth could be achieved after culturing using a 3D approach. These findings suggested that ADSCs may provide a novel promising therapeutic approach for the treatment of patients with liver cancer, and the 3D culture method may provide a novel approach to explore the association between ADSCs and cancer.

\section{Introduction}

Stem cell therapy has been considered a potential regenerative strategy for the treatment of various diseases, including diabetes, Parkinson's disease, cancer and other chronic long-standing conditions $(1,2)$. At present, various cell types have been used in stem cell therapy, including embryonic stem cells, induced pluripotent stem cells, hematopoietic stem cells (HSCs) and mesenchymal stem cells (MSCs) (3-6). Besides the application of HSCs for the treatment of blood diseases, the majority of studies regarding stem cell therapy have focused on MSCs. MSCs are adult stem cells that can be isolated form bone marrow, adipose tissue, umbilical cord, heart tissue and skin $(7,8)$. MSCs possess not only the ability to differentiate into numerous mesenchymal lineages $(9,10)$, but may also regulate the immune response and repair injured tissues without ethical concerns (11).

Compared with other sources of MSCs, adipose-derived stem cells (ADSCs) are abundant and easier to obtain. Furthermore, ADSCs have been reported to lack major histocompatibility complex-II expression, and are more potent than bone marrow-derived stem cells (BMSCs) in suppressing dendritic cell differentiation $(12,13)$. These findings suggested that ADSCs possess better immunoregulatory abilities compared with BMSCs. ADSCs-based therapy has been clinically used to treat perianal fistula (14-18), postoperative enterocutaneous fistula (19), osteoarthritis (20), acute ischemic stroke (21) and idiopathic pulmonary fibrosis (22). Furthermore, ADSCs-based therapy may be used to treat cardiovascular disease, liver disease and neurological disorders in preclinical trails (23-25). 
Although ADSCs-based therapy has been widely used for several diseases in preclinical and clinical investigations, the long-term safety, particularly tumorigenicity, is a major barrier for clinical translation of ADSCs-based therapy. Therefore, the association between ADSCs and cancer has attracted extensive interest in stem cell therapy. Numerous studies have indicated that ADSCs may promote tumor progression. For example, Chu et al reported that ADSCs could promote epithelial ovarian cancer growth and metastasis (26). Muehlberg et al revealed that ADSCs promoted breast cancer growth and metastasis (27). Conversely, other studies have suggested that ADSCs may suppress bladder tumor growth and inhibit breast cancer metastasis $(28,29)$. Therefore, the tumorigenicity of ADSCs remains controversial.

It is widely recognized that various cytokines and chemokines that are secreted from ADSCs may affect the proliferation of tumor cells. However, the levels of cytokines and chemokines secreted from ADSCs are closely associated with culture conditions $(30,31)$. For example, Bhang et al demonstrated that ADSCs cultured under three-dimensional (3D) culture conditions could produce higher concentrations of angiogenic and/or anti-apoptotic factors, including vascular endothelial growth factor, fibroblast growth factor-2, hepatocyte growth factor and C-X-C motif chemokine ligand 12 compared with cells cultured under two-dimensional (2D) conditions (32). In addition, Yang et al demonstrated that the 3D culture method could enhance the activity of ADSCs and increase the autophagic response upon hydrogen peroxide $\left(\mathrm{H}_{2} \mathrm{O}_{2}\right)$ treatment compared with the $2 \mathrm{D}$ culture method (33). Tian et al revealed that human MSCs inhibited proliferation of cancer cells in vitro, whereas they enhanced tumor formation and growth in vivo (34), thus indicating the dual effects of MSCs on the same tumor. Therefore, whether ADSCs serve a protumorigenic or anti-tumorigenic role in tumor growth depends on the in vivo or in vitro growing conditions of ADSCs. Using an appropriate culture method, which closely mimics in vivo conditions, may be of great benefit to illustrate the association between cancer and ADSCs. In order to better understand how ADSCs affect tumors, the present study used different culture methods, including 2D culture method, sphere culture method and AlgiMatrix ${ }^{\mathrm{TM}}$ 3D culture method, to investigate whether cultured ADSCs may promote or inhibit the growth of liver cancer cells, and to explore the underlying mechanisms.

\section{Materials and methods}

Animals and ethics approval. A total of 5 adult male Sprague-Dawley (SD) rats (weight, 180-200 g; age, 7-8 week old) were obtained from the Center for Animal Experiments of Fujian Medical University (Fuzhou, China; license no. SCXKmin2012-0002). The rats were housed at a constant temperature $\left(22 \pm 2^{\circ} \mathrm{C}\right)$, with $60 \%$ relative humidity, under a 12-h light/dark cycle. The rats had ad libitum access to food and autoclaved water. The present study was approved by the Animal Ethics Committee of Fuzhou General Hospital (Fuzhou, China).

Cell culture. Rat ADSCs were derived from subcutaneous adipose tissues according to the protocol described in our previous study (35). Briefly, following anesthetization of the male SD rats $(n=5)$ using pentobarbital sodium $(40 \mathrm{mg} / \mathrm{kg}$;
Merck \& Co., Inc., Whitehouse Station, NJ, USA), adipose tissues $(\sim 3 \times 1.5 \times 0.5 \mathrm{~cm})$ were scraped from the subcutaneous inguinal region, cut into small pieces $(\sim 0.1 \times 0.1 \times 0.1 \mathrm{~mm})$, and digested with $0.1 \%$ type I collagenase (Sigma-Aldrich; Merck KGaA, Darmstadt, Germany) at $37^{\circ} \mathrm{C}$ for 60 min with gentle agitation. Subsequently, the digested tissue was filtered through a $100-\mu \mathrm{m}$ cell strainer, centrifuged at $400 \mathrm{x} \mathrm{g}$ for 5 min and washed twice with PBS (HyClone; GE Healthcare Life Sciences, Logan, UT, USA). The cell pellet was then suspended in expanding medium comprising $\alpha$-modified Eagle's medium ( $\alpha$-MEM; HyClone; GE Healthcare Life Sciences) supplemented with $20 \%$ fetal bovine serum (FBS), penicillin $(100 \mathrm{U} / \mathrm{ml})$ and streptomycin $(100 \mu \mathrm{g} / \mathrm{ml})$ (all from Gibco; Thermo Fisher Scientific, Inc., Waltham, MA, USA). The cell suspension was transferred into a 6-well plate (Corning Inc., Corning, NY, USA) at a density of $1 \times 10^{6} / \mathrm{ml}$ and was incubated at $37^{\circ} \mathrm{C}$ in an atmosphere containing $5 \% \mathrm{CO}_{2}$. After $24 \mathrm{~h}$, the adherent cells were further expanded in complete medium comprising $\alpha$-MEM, $10 \% \mathrm{FBS}, 100 \mathrm{U} / \mathrm{ml}$ penicillin and $100 \mu \mathrm{g} /$ $\mathrm{ml}$ streptomycin. ADSCs from passage 3 or 4 were used in the present study. Liver cancer cell lines, including the Hcclm3 human hepatocellular carcinoma (HCC) cell line and the HepG2 human hepatoblastoma cell line (36), were purchased from the Cell Bank of the Chinese Academy of Sciences (Shanghai, China), and were cultured in complete medium comprising Dulbecco's modified Eagle's medium (DMEM; Gibco; Thermo Fisher Scientific, Inc.) supplemented with $10 \%$ FBS, $100 \mathrm{U} / \mathrm{ml}$ penicillin and $100 \mu \mathrm{g} / \mathrm{ml}$ streptomycin. All cells were incubated in a humidified atmosphere containing $5 \% \mathrm{CO}_{2}$ at $37^{\circ} \mathrm{C}$.

Preparation of ADSCs-conditioned medium (CM). To collect the 2D-ADSCs-CM, sphere-ADSCs-CM and 3D-ADSCs-CM, rat $\mathrm{ADSCs}$ were seeded at a density of $2 \times 10^{5}$ cells/well into conventional 6-well plates, 6-well non-adherent plates (Corning, Inc.) and AlgiMatrix ${ }^{\mathrm{TM}}$ 3D culture system 6-well plates (Gibco; Thermo Fisher Scientific, Inc.), respectively. The cells were cultured with StemPro ${ }^{\circledR}$ MSC SFM CTS ${ }^{\text {TM }}$ medium (MSC medium; Gibco; Thermo Fisher Scientific, Inc.). All cells were incubated in a humidified atmosphere containing $5 \% \mathrm{CO}_{2}$ at $37^{\circ} \mathrm{C}$. After $48 \mathrm{~h}$, the medium was collected, filtered through a $0.22 \mu \mathrm{m}$ filter and stored at $-80^{\circ} \mathrm{C}$.

Cell viability assay. In order to observe the effects of ADSCs on tumor cell proliferation, Hcclm 3 and HepG 2 cells were cultured in 96-well plates at a density of $5 \times 10^{3}$ cells/well with $150 \mu \mathrm{l}$ complete medium, at $37^{\circ} \mathrm{C}$ in an atmosphere containing $5 \% \mathrm{CO}_{2}$ for $24 \mathrm{~h}$. Subsequently, the cell culture medium of each well was replaced with $100 \mu \mathrm{l}$ ADSC-CM (5x10 ${ }^{4}$ ADSCs/ ml MSC medium); cells incubated with $100 \mu \mathrm{l}$ MSC medium were used as the negative control. Following a $48 \mathrm{~h}$ incubation at $37^{\circ} \mathrm{C}$, cell viability was determined using the Cell Counting kit-8 (CCK-8) cell proliferation kit (Dojindo Molecular Technologies, Inc., Kumamoto, Japan) according to the manufacturer's protocol. A microplate reader (SpectraMax M5; Molecular Devices, LLC, Sunnyvale, CA, USA) was used to measure the absorbance of the solution in each well at $450 \mathrm{~nm}$. All experiments were performed in triplicate.

Cell apoptosis assay. Flow cytometry was used to assess cell apoptosis. Briefly, Hcclm3 and HepG2 cells were cultured 
in 6-well plates at a density of $1 \times 10^{5}$ cells/well for $24 \mathrm{~h}$. Subsequently, the culture medium was discarded and replaced

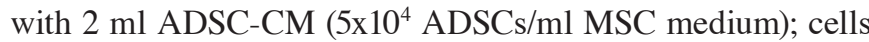
incubated with $2 \mathrm{ml} \mathrm{MSC}$ medium were used as the negative control. After $48 \mathrm{~h}$, tumor cells from all groups were further incubated with $1 \% \mathrm{H}_{2} \mathrm{O}_{2}$ for $10 \mathrm{~min}$ at room temperature, in order to induce cell apoptosis of some tumor cells. Tumor cells were then collected and cell apoptosis was analyzed using the Annexin V-fluorescein isothiocyanate apoptosis assay kit (Dojindo Molecular Technologies, Inc.) according to the manufacturer's protocol. Finally, the labeled cells were assessed using a FACSVerse flow cytometer (BD Biosciences, Franklin Lakes, NJ, USA), and all raw data were analyzed with ModFit LT v2.0 software (Verity Software House, Inc., Topsham, ME, USA). All experiments were performed in triplicate.

Scratch wound healing assay. Hcclm 3 and HepG2 cells $\left(1.5 \times 10^{5}\right.$ cells/well) were seeded into 24 -well plates and allowed to adhere overnight. After reaching 80-90\% confluence, a 'reference line' was scratched at the bottom of the plate using a sterile $10 \mu \mathrm{l}$ pipette tip. Subsequently, cells were washed three times with PBS, and were further incubated with $1 \mathrm{ml}$ ADSC-CM (1.5x10 $\mathrm{ADSCs} / \mathrm{ml} \mathrm{MSC} \mathrm{medium)} \mathrm{to}$ observe the migration of liver cancer cells into the cell-free area. Photomicrographs of cells migrating across the 'reference line' were captured from different fields following each treatment at 0 and $48 \mathrm{~h}$ with an inverted microscope (Zeiss GmbH, Jena, Germany).

Cell adhesion assay. To observe the effects of ADSCs on liver cancer cell adhesion, 96-well microplates were pretreated with $50 \mu \mathrm{l}$ fibronectin $(20 \mu \mathrm{g} / \mathrm{ml}$; BD Biosciences). Liver cancer cells $\left(4 \times 10^{3}\right.$ cells/well) were suspended in $100 \mu \mathrm{l}$ ADSCs-CM $\left(4 \times 10^{4}\right.$ ADSCs/ml MSC medium) and were added to the pretreated 96-well plates. At the indicated time-point, the attached cells were fixed in absolute methanol and stained with $10 \%$ crystal violet at room temperature for $30 \mathrm{~min}$. Subsequently, the cells were washed with PBS and images were captured using an inverted microscope (Zeiss $\mathrm{GmbH}$ ). To further quantify the adhesive capabilities of the cells, crystal violet-stained cells were treated with $33 \%$ acetic acid (150 $\mu \mathrm{l} /$ well) in PBS for $5 \mathrm{~min}$ at room temperature. A microplate reader (SpectraMax M5; Molecular Devices, LLC) was used to measure the absorbance of the solution in each well at $570 \mathrm{~nm}$. All experiments were performed in triplicate.

Cell migration and invasion assays. A cell migration assay was conducted using Transwell units (no. 3422; Corning Inc.) and cell invasion was assessed using Matrige ${ }^{\circledR}$-coated Transwell units (no. 354480; BD Biosciences) on a 24-well plate. For tumor cell migration and invasion assays, $1 \times 10^{5}$ liver cancer cells were cultured in the upper compartment of the Transwell units with $200 \mu \mathrm{l}$ ADSCs-CM $\left(5 \times 10^{5}\right.$ ADSCs $/ \mathrm{ml}$ MSC medium), whereas the lower compartment of the units was filled with DMEM supplemented with $10 \%$ FBS. After $48 \mathrm{~h}$, the filters were fixed with $4 \%$ paraformaldehyde at room temperature for $20 \mathrm{~min}$ and stained with $10 \%$ crystal violet at room temperature for $30 \mathrm{~min}$. Cells on the upper surface of the Transwell units were removed using a cotton swab, and cells that had passed through the lower surface of the filter were counted under an inverted microscope (Zeiss $\mathrm{GmbH}$ ) in five different fields at a magnification of x200.

Reverse transcription-quantitative polymerase chain reaction (RT-qPCR) analysis. Following incubation with ADSCs-CM for $48 \mathrm{~h}$, total RNA was isolated from liver cancer cells using TRIzol ${ }^{\circledR}$ reagent (Invitrogen; Thermo Fisher Scientific, Inc.). Subsequently, mRNA was reverse transcribed to cDNA using a Transcriptor First Strand cDNA Synthesis kit (Roche Applied Science, Mannheim, Germany) according to the manufacturer's protocol. qPCR analysis was performed using the ABI StepOnePlus Real-Time PCR system (Applied Biosystems; Thermo Fisher Scientific, Inc.) with q-PCR Master Mix (Toyobo Life Science, Osaka, Japan). Cycling conditions were as follows: initial denaturation at $95^{\circ} \mathrm{C}$ for $2 \mathrm{~min}$; 40 cycles at $95^{\circ} \mathrm{C}$ for $15 \mathrm{sec}, 60^{\circ} \mathrm{C}$ for $30 \mathrm{sec}$ and $70^{\circ} \mathrm{C}$ for $30 \mathrm{sec}$. The PCR primer sequences were as follows: E-cadherin, forward CGA GAGCTACACGTTCACGG, reverse GGGTGTCGAGGG AAAAATAGG; N-cadherin, forward AGCCAACCTTAA CTGAGGAGT, reverse GGCAAGTTGATTGGAGGGATG; vimentin, forward GACGCCATCAACACCGAGTT, reverse CTTTGTCGTTGGTTAGCTGGT; zinc finger E-box-binding homeobox 1 (Zeb1), forward CTACAACAACAAGACACT GCTGT, reverse TGTTCTTTCAGAGAGGTAAAGCG; and $\beta$-actin, forward ATAGCACAGCCTGGATAGCAACGTAC and reverse CACCTTCTACAATGAGCTGCGTGTG. Target gene expression was normalized to that of $\beta$-actin. Relative gene expression was calculated using the $2^{-\Delta \Delta \mathrm{Cq}}$ method (37). Experiments were independently repeated three times.

Western blotting. After incubating with ADSCs-CM for $48 \mathrm{~h}$, liver cancer cells were lysed in ice-cold radioimmunoprecipitation assay buffer [0.5 M Tris- $\mathrm{HCl}(\mathrm{pH} 7.4), 1.5 \mathrm{M} \mathrm{NaCl}$, $2.5 \%$ deoxycholic acid, 10\% NP-40 and $10 \mathrm{mM}$ EDTA] with protease inhibitor cocktail (Roche Diagnostics, Indianapolis, IN, USA). Protein quantification was performed by bicinchoninic acid assay, and equal amounts of protein lysate $(40 \mu \mathrm{g})$ were separated by $12 \%$ SDS-PAGE. Proteins were then transferred to nitrocellulose membranes in transfer buffer [12 mM Tris base, $96 \mathrm{mM}$ glycine (pH 8.3) and 15\% methanol]. Subsequently, the membranes were blocked at room temperature for $2 \mathrm{~h}$ in Tris-buffered saline containing $0.1 \%$ Tween (TBST) containing $5 \%$ bovine serum albumin (Sigma-Aldrich; Merck KGaA), and were probed with E-cadherin (3195), vimentin (5741), $\mathrm{N}$-cadherin (13116) and Zeb1 (3396) antibodies (1:500 dilution; all from Cell Signaling Technology, Inc., Danvers, MA, USA) and $\beta$-actin antibody (1:5,000 dilution; Beijing TransGen Biotech Co., Ltd., Beijing, China) overnight at $4^{\circ} \mathrm{C}$. The membranes were washed three times with TBST, and were then incubated with the appropriate horseradish peroxidase-conjugated secondary antibody (1:8,000 dilution; sc-2004; Santa Cruz Biotechnology, Inc., Dallas, TX, USA) for $1 \mathrm{~h}$ at room temperature. Finally, the protein expression levels were detected by enhanced chemiluminescence using supersignal west pico chemiluminescent substrate (Pierce; Thermo Fisher Scientific, Inc.) and visualized by autoradiography using Image Lab $^{\mathrm{TM}} 4.1$ software (Bio-Rad, Hercules, CA, USA).

Statistical analysis. All quantitative data are expressed as the means \pm standard deviation. All statistical analyses were 
A

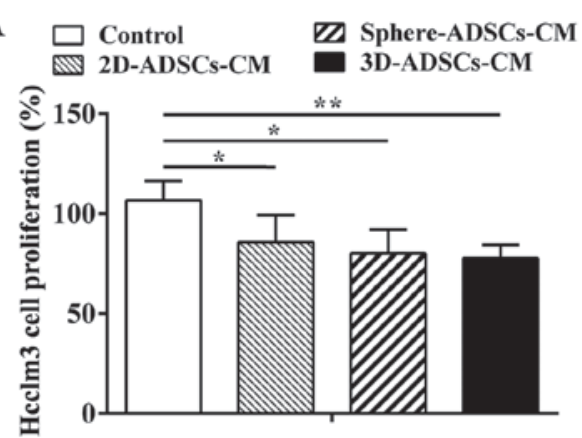

B

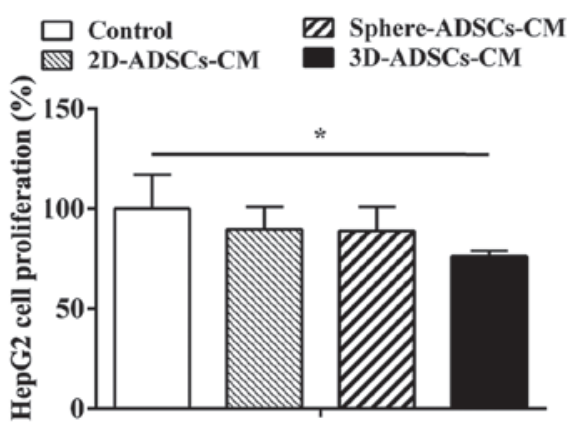

Figure 1.ADSCs-CM inhibits liver cancer cell proliferation. Proliferation of (A) Hcclm3 and (B) HepG2 cells after culturing with ADSCs-CM. Control, untreated liver cancer cells; 2D-ADSCs-CM, liver cancer cells treated with 2D-ADSCs-CM; sphere-ADSCs-CM, liver cancer cells treated with sphere-ADSCs-CM; 3D-ADSCs-CM, liver cancer cells treated with 3D-ADSCs-CM. For all groups, $\mathrm{n}=4$. ${ }^{*} \mathrm{P}<0.05$ and ${ }^{* *} \mathrm{P}<0.01$. 2D, two-dimensional; 3D, three-dimensional; ADSCs-CM, adipose tissue-derived stem cells-conditioned medium.

performed using SPSS 16.0 (SPSS, Inc., Chicago, IL, USA). Multiple comparisons were analyzed by one-way analysis of variance, followed by LSD multiple comparison test. $\mathrm{P}<0.05$ was considered to indicate a statistically significant difference.

\section{Results}

ADSCs-CM inhibits proliferation of liver cancer cells. To investigate whether ADSCs affect the proliferation of tumor cells, HepG2 and Hcclm3 cells were cultured with three various types of ADSCs-CM: 2D-ADSCs-CM, sphere-ADSCs-CM and 3D-ADSCs-CM for $48 \mathrm{~h}$. Cell proliferation of treated tumor cells was then evaluated using the CCK-8 assay. The results indicated that the proliferation of Hcclm3 cells was inhibited following culture with 2D-ADSCs-CM, sphere-ADSCs-CM and 3D-ADSCs-CM (Fig. 1A); however, despite a slight decrease after culturing with 2D-ADSCs-CM or sphere-ADSCs-CM, the proliferation of HepG2 cells was only significantly inhibited when cultured with 3D-ADSCs-CM (Fig. 1B). In addition, the proliferation of liver cancer cells was more markedly reduced after culturing with 3D-ADSCs-CM compared with sphere-ADSCs-CM or 2D-ADSCs-CM; however, there was no significant difference detected in cell proliferation among the three groups cultured with various types of ADSCs-CM (Fig. 1). These results suggested that ADSCs-CM, particularly 3D-ADSCs-CM, could significantly inhibit liver cancer cell proliferation, and the 3D culture method could partly accelerate the inhibitory effects of ADSCs on liver cancer cell proliferation.

ADSCs-CM promotes apoptosis of liver cancer cells. In order to evaluate the effects of ADSCs on the apoptosis of Hcclm3 and HepG2 cells, the apoptotic rate of tumor cells was determined following treatment with ADSCs-CM by flow cytometry. Compared with the control liver cancer cells, the proportion of apoptotic liver cancer cells was increased after culturing with ADSCs-CM (Fig. 2A and B). To further determine the apoptotic rate of liver cancer cells, data were quantified by analyzing the percentage of Annexin V-positive cells. According to these results, the liver cancer cell apoptotic rate was significantly increased after culturing with ADSCs-CM compared with the control group. In addition, the highest cell apoptotic rate was observed in liver cancer cells treated with sphere-ADSCs-CM or 3D-ADSCs-CM compared with those treated with 2D-ADSCs-CM (Fig. 2C and D). These results clearly indicated that ADSCs-CM may significantly promote the apoptosis of liver cancer cells, and apoptosis of liver cancer cells could be enhanced by culturing with sphere-ADSCs-CM or 3D-ADSCs-CM. In addition, the present data demonstrated that more significantly increased apoptosis of liver cancer cells could be achieved after culturing with 3D-ADSCs-CM compared with sphere-ADSCs-CM in HepG2 cells (Fig. 2C and D). These findings suggested that the $3 \mathrm{D}$ culture method may result in the enhancement of ADSCs-induced HepG2 cell apoptosis.

ADSCS-CM inhibits motility and adhesive capacity of liver cancer cells. The present study used the wound healing assay to evaluate motility of tumor cells after culturing with ADSCs-CM. Compared with the control liver cancer cells, motility of liver cancer cells was reduced after culturing with ADSCs-CM (Fig. 3A and B). To further determine liver cancer cell motility, the distance of cell motility was quantified. As shown in Fig. 3C and D, it was confirmed that liver cancer cell motility was decreased after culturing with ADSCs-CM; in particular, liver cancer cells exhibited the lowest cell motility after culturing with 3D-ADSCs-CM compared with 2D-ADSCs-CM or sphere-ADSCs-CM. However, when liver cancer cells were cultured with sphere-ADSCs-CM, there was no significant difference compared with those cultured with 2D-ADSCs-CM. On the basis of these results, the present study suggested that ADSCs-CM may significantly inhibit liver cancer cell motility, and the 3D culture method could accelerate the inhibitory effects of ADSCs on liver cancer cell motility.

Due to the inhibitory effects of ADSCs on liver cancer cell motility, the present study further evaluated the effects of ADSCs on the adhesive capacity of liver cancer cells. Hcclm3 and HepG2 cells were cultured with ADSCs-CM in fibronectin-coated plates for 20,40 and $60 \mathrm{~min}$; adhesive cells were examined by crystal violet staining. Although no significant difference was observed between the control and ADSCs-CM-treated liver cancer cells after 20 or $40 \mathrm{~min}$ (data not shown), the number of adhesive cells was decreased following incubation with ADSCs-CM for 60 min compared with the control liver cancer cells (Fig. 3E-G). In addition, a more obvious inhibition of liver cancer cell adhesion was detected after culturing with 3D-ADSCs-CM compared with sphere-ADSCs-CM or 2D-ADSCs-CM; however, no significant 


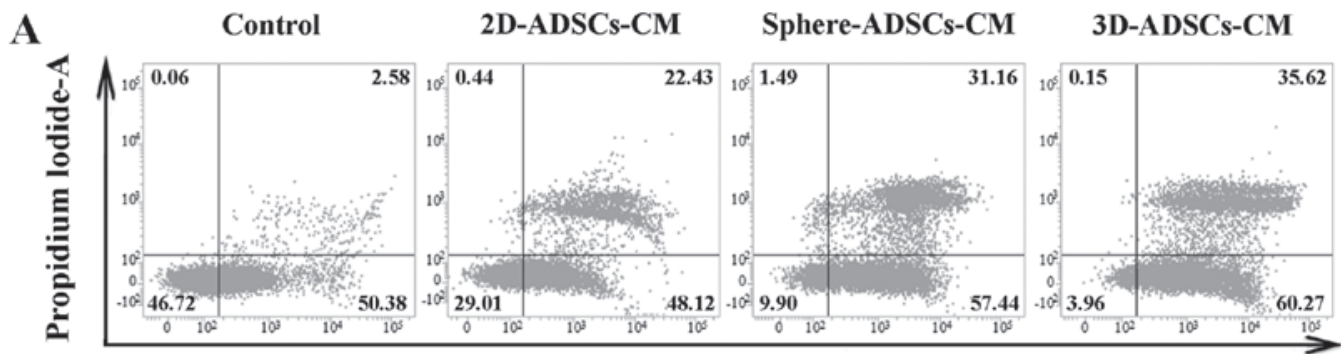

Hcclm3 annexin V FITC-A

B

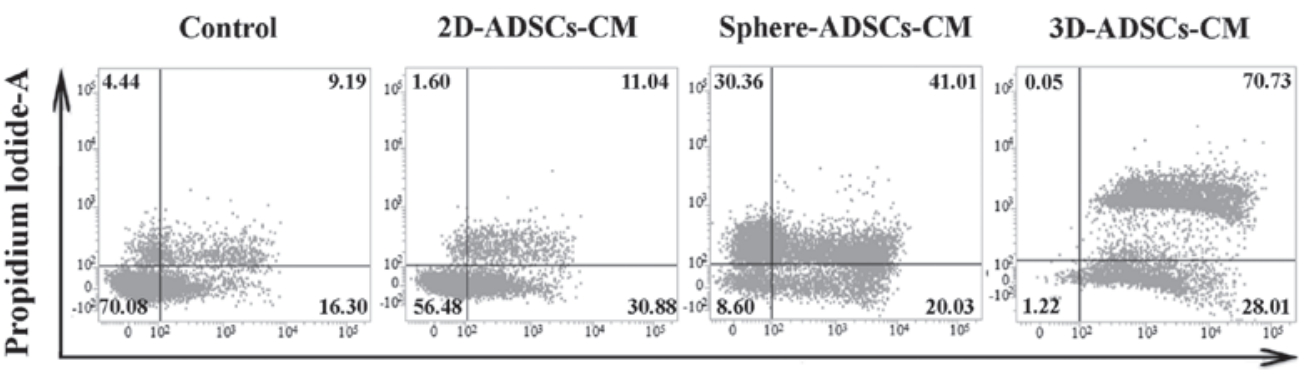

HepG2 annexin V FITC-A

C

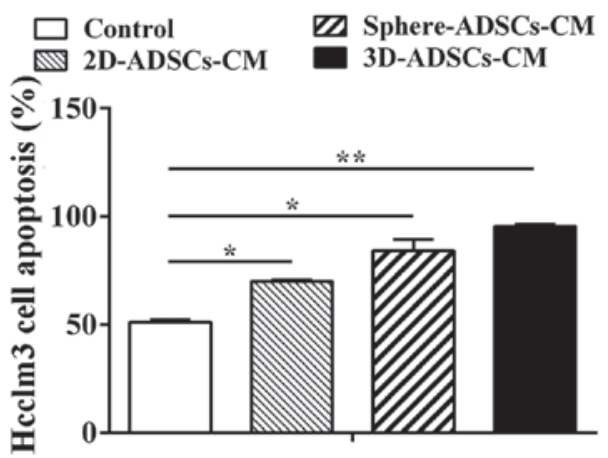

D

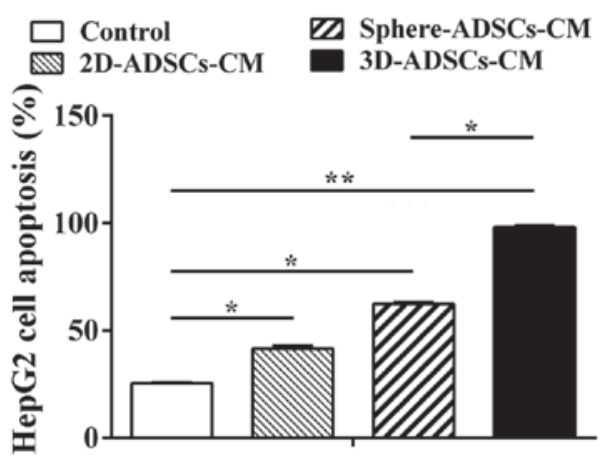

Figure 2. ADSCs-CM promotes liver cancer cell apoptosis. Proportion of (A) Hcclm3 and (B) HepG2 apoptotic cells after culturing with ADSCs-CM. Apoptotic rate of (C) Hcclm3 and (D) HepG2 cells after culturing with ADSCs-CM. Control, untreated liver cancer cells; 2D-ADSCs-CM, liver cancer cells treated with 2D-ADSCs-CM; sphere-ADSCs-CM, liver cancer cells treated with sphere-ADSCs-CM; 3D-ADSCs-CM, liver cancer cells treated with 3D-ADSCs-CM. For all groups, $\mathrm{n}=3 .{ }^{*} \mathrm{P}<0.05$ and ${ }^{* *} \mathrm{P}<0.01$. 2D, two-dimensional; 3D, three-dimensional; ADSCs-CM, adipose tissue-derived stem cells-conditioned medium; FITC, fluorescein isothiocyanate.

difference was detected among the three ADSCs-CM groups. These data indicated that ADSCs could inhibit liver cancer cell adhesion, and the 3D culture method could partly enhance the inhibitory effects of ADSCs on liver cancer cell adhesion.

ADSCs-CM suppresses migration and invasion of liver cancer cells. To confirm the effects of ADSCs on migration of tumor cells, liver cancer cells were cultured with ADSCs-CM in Transwell units for $48 \mathrm{~h}$; the liver cancer cells that penetrated the membrane were examined by crystal violet staining. The results indicated that the number of liver cancer cells that migrated across the membrane was significantly decreased after culturing with ADSCs-CM compared with the control liver cancer cells (Fig. 4A-C), thus indicating that ADSCs may suppress migration of liver cancer cells. The present results also demonstrated that liver cancer cell migration was more significantly decreased after culturing with 3D-ADSCs-CM or sphere-ADSCs-CM compared with 2D-ADSCs-CM; in addition, the lowest rate of liver cancer cell migration was observed after culturing with 3D-ADSCs-CM compared with sphere-ADSCs-CM (Fig. 4B and C). These results indicated that the sphere or $3 \mathrm{D}$ culture methods may enhance the inhibitory effects of ADSCs on liver cancer cell migration, and the 3D culture method is considered the best candidate to enhance the suppressive effects of ADSCs on liver cancer cell migration.

Based on the observation that ASDCs-CM may suppress liver cancer cell migration, the present study aimed to determine whether ADSCs-CM could affect the invasion of liver cancer cells. Hcclm3 and HepG2 cells were cultured with ADSCs-CM in Matrigel ${ }^{\circledR}$-coated Transwell units for $48 \mathrm{~h}$, and liver cancer cells that penetrated the membrane were examined by crystal violet staining. Compared with the control liver cancer cells, cell invasion was significantly inhibited after culturing with ADSCs-CM (Fig. 4D-F), thus suggesting that ADSCs may suppress the invasion of liver cancer cells. In addition, the present results indicated that the lowest rate of liver cancer cell 
A

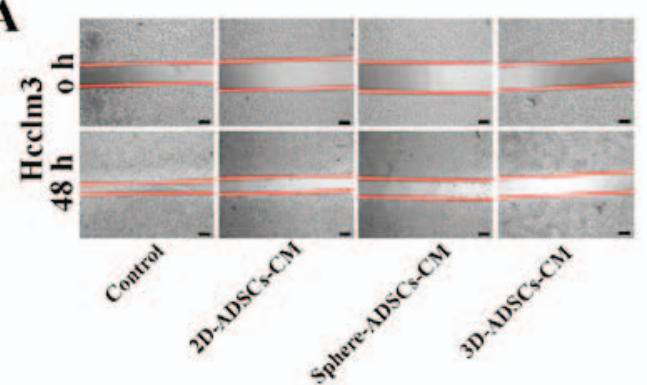

C

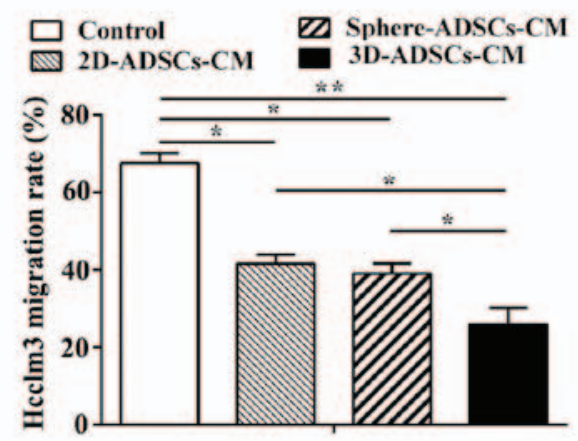

B

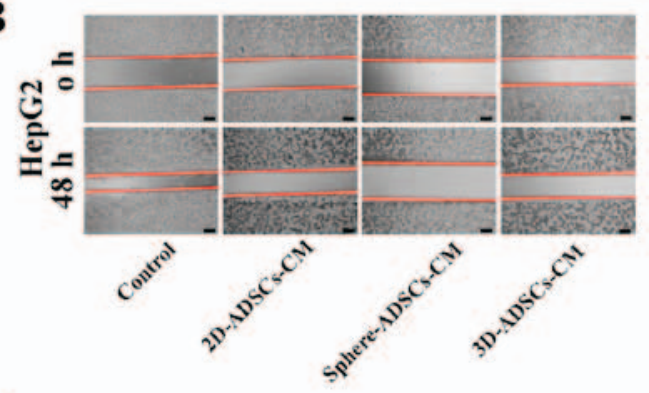

D
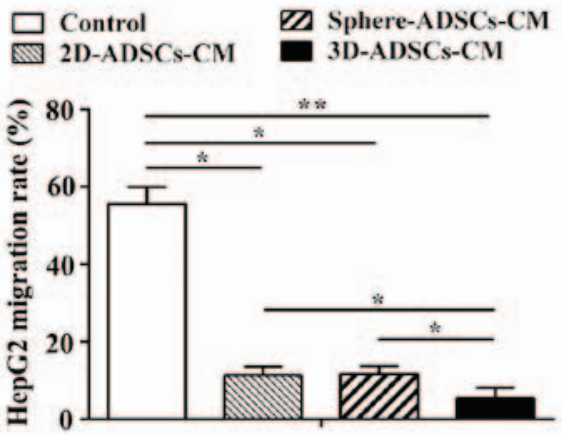

$\mathbf{E}$

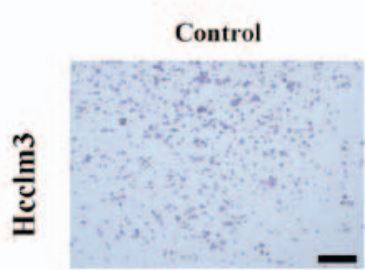

2D-ADSCs-CM

Sphere-ADSCs-CM

3D-ADSCs-CM

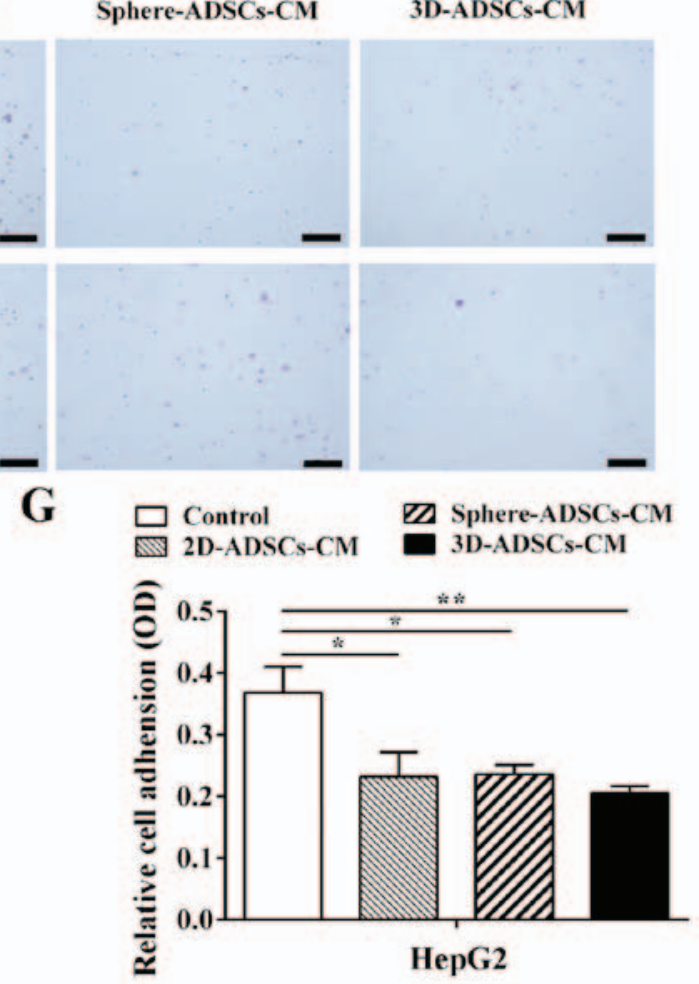

Figure 3. ADSCs-CM inhibits motility and adhesive capacity of liver cancer cells. Motility of (A) Hcclm3 and (B) HepG2 cells after culturing with ADSCs-CM (x100 magnification; scale bar, $50 \mu \mathrm{m}$ ). Quantification of (C) Hcclm3 and (D) HepG2 cell migration after culturing with ADSCs-CM. (E) Number of adhesive cells after culturing with ADSCs-CM for 60 min (x50 magnification; scale bar, $200 \mu \mathrm{m}$ ). Quantification of (F) Hcclm3 and (G) HepG2 cell adhesion after culturing with ADSCs-CM. Control, untreated liver cancer cells; 2D-ADSCs-CM, liver cancer cells treated with 2D-ADSCs-CM; sphere-ADSCs-CM, liver cancer cells treated with sphere-ADSCs-CM; 3D-ADSCs-CM, liver cancer cells treated with 3D-ADSCs-CM. For all groups, $\mathrm{n}=3$. ${ }^{*} \mathrm{P}<0.05$ and ${ }^{* * *} \mathrm{P}<0.01 .2 \mathrm{D}$, two-dimensional; 3D, three-dimensional; ADSCs-CM, adipose tissue-derived stem cells-conditioned medium; OD, optical density.

invasion could be achieved after culturing with 3D-ADSCs-CM compared with 2D-ADSCs-CM. Furthermore, although the difference was not statistically significant, a slight decrease in liver cancer cell invasion could be observed after culturing with 3D-ADSCs-CM compared with sphere-ADSCs-CM. Liver cancer cell invasion was also not significantly decreased after culturing with sphere-ADSCs-CM compared with 2D-ADSCs-CM (Fig. 4D-F). Therefore, these results suggested that the inhibitory effects of ADSCs on liver cancer cell invasion could be enhanced by the $3 \mathrm{D}$ or sphere culture method, but not by the sphere culture method.

ADSCs-CM suppresses epithelial-mesenchymal transition (EMT) in liver cancer cells. In order to further explore 

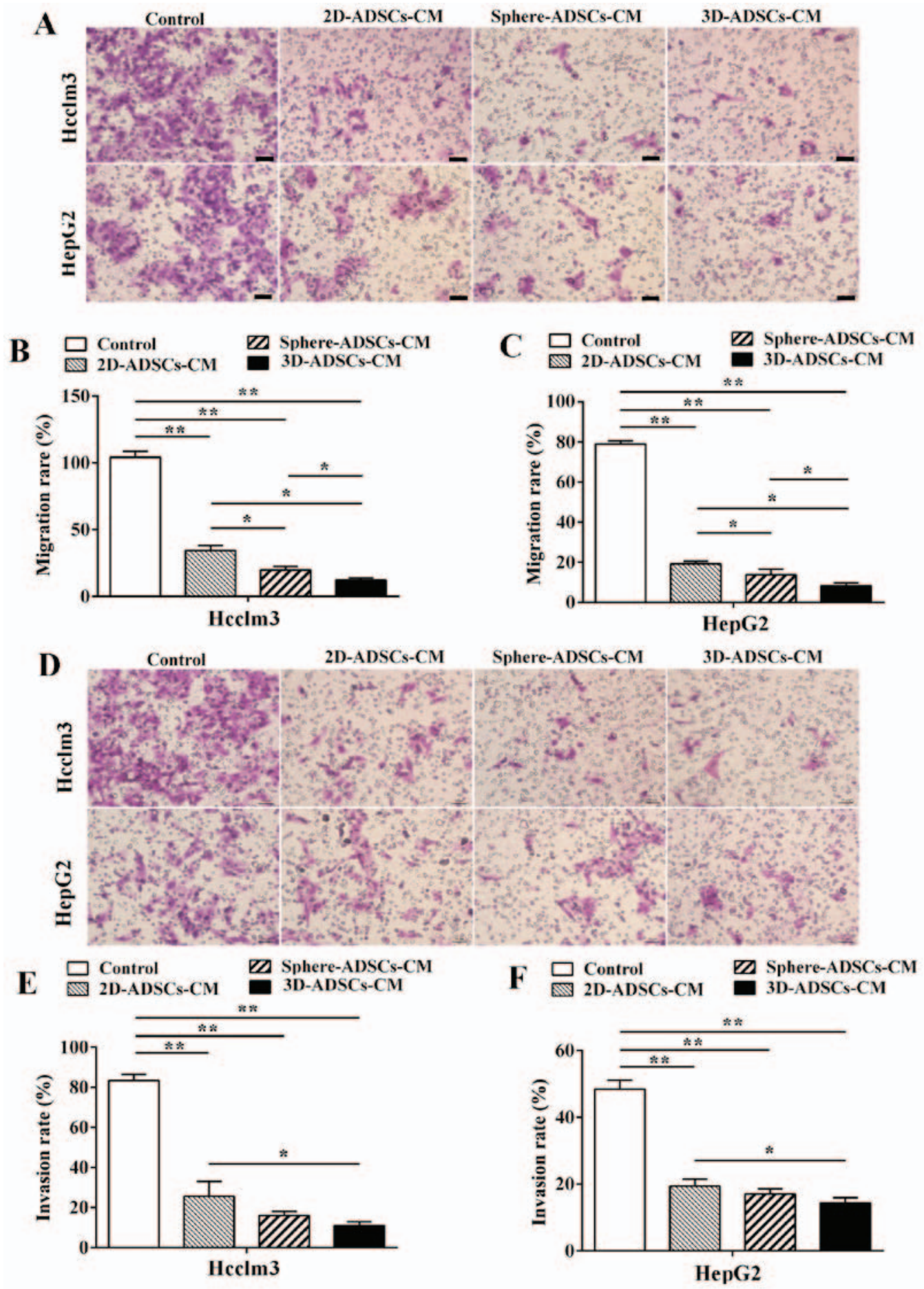

Figure 4. ADSCs-CM suppresses migration and invasion of liver cancer cells. (A) Migration of liver cancer cells after culturing with ADSCs-CM (x100 magnification; scale bar, $100 \mu \mathrm{m}$ ). Quantification of (B) Hcclm3 and (C) HepG2 cell migration after culturing with ADSCs-CM. (D) Invasion of liver cancer cells was observed after culturing with ADSCs-CM (x100 magnification; scale bar, $100 \mu \mathrm{m}$ ). Quantification of (E) Hcclm3 and (F) HepG2 cell migration after culturing with 3D-ADSCs-CM. Control, untreated liver cancer cells; 2D-ADSCs-CM, liver cancer cells treated with 2D-ADSCs-CM; sphere-ADSCs-CM, liver cancer cells treated with sphere-ADSCs-CM; 3D-ADSCs-CM, liver cancer cells treated with 3D-ADSCs-CM. For all groups, $\mathrm{n}=3$. ${ }^{*} \mathrm{P}<0.05$ and ${ }^{* *} \mathrm{P}<0.01 .2 \mathrm{D}$, two-dimensional; 3D, three-dimensional; ADSCs-CM, adipose tissue-derived stem cells-conditioned medium

the underlying mechanisms by which ADSCs inhibit the migration and invasion of liver cancer cells, the present study investigated whether ADSCs-CM had an effect on EMT of liver cancer cells, since EMT serves an important role in the progression of cancer invasion and metastasis (38). Hcclm 3 and HepG2 cells were harvested after culturing with ADSCs-CM for $48 \mathrm{~h}$, and EMT markers were examined by
RT-qPCR analysis. As shown in Fig. 5, ADSCs-CM significantly increased the mRNA expression levels of E-cadherin, and downregulated the mRNA expression levels of $\mathrm{N}$-cadherin, vimentin and Zeb1 in Hcclm3 and HepG2 cells, thus indicating that ADSCs-CM could inhibit liver cancer cell migration and invasion by downregulating EMT at the transcriptional level. 
A

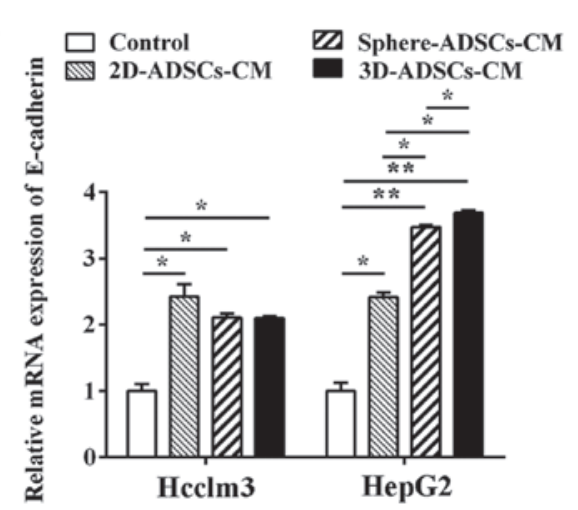

C

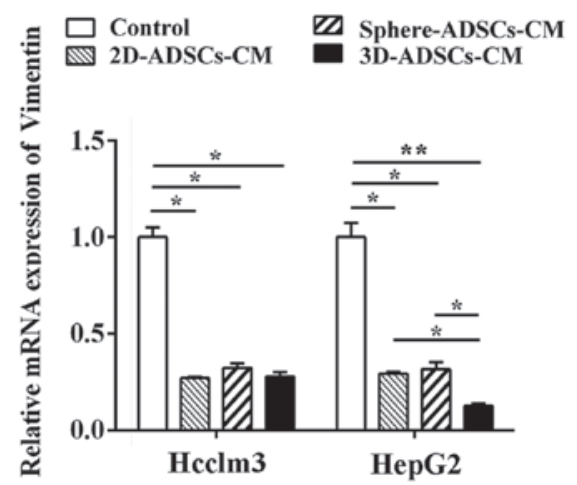

B

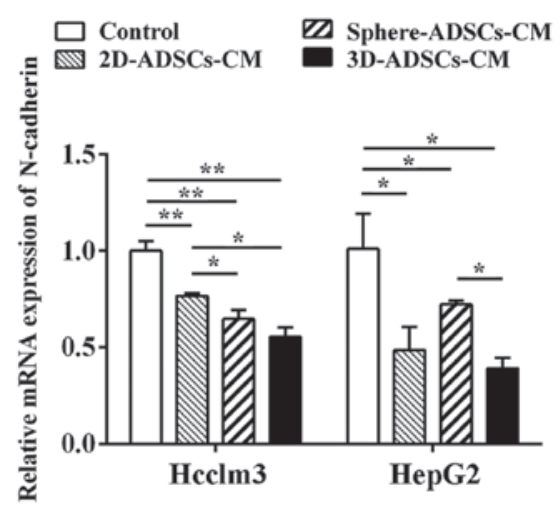

D

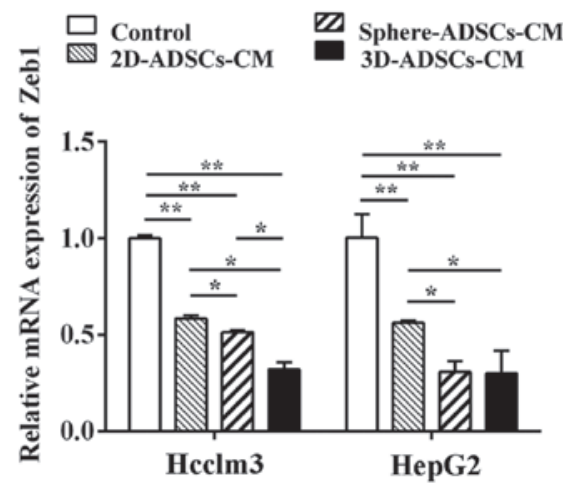

Figure 5. ADSCs-CM downregulates the mRNA expression levels of EMT markers in liver cancer cells. mRNA expression levels of (A) E-cadherin, (B) N-cadherin, (C) vimentin and (D) Zeb1 in liver cancer cells after culturing with ADSCs-CM. Control, untreated liver cancer cells; 2D-ADSCs-CM, liver cancer cells treated with 2D-ADSCs-CM; sphere-ADSCs-CM, liver cancer cells treated with sphere-ADSCs-CM; 3D-ADSCs-CM, liver cancer cells treated with 3D-ADSCs-CM. For all groups, $\mathrm{n}=3$. ${ }^{*} \mathrm{P}<0.05$ and ${ }^{* *} \mathrm{P}<0.01$. 2D, two-dimensional; 3D, three-dimensional; ADSCs-CM, adipose tissue-derived stem cells-conditioned medium; EMT, epithelial-mesenchymal transition; Zeb1, zinc finger E-box-binding homeobox 1.

The present results also indicated that increased expression of E-cadherin mRNA (in HepG2 cells), and reduced mRNA expression of $\mathrm{N}$-cadherin (in Hcclm 3 cells) and Zeb1 (in Hcclm3 and HepG2 cells), was observed after culturing with sphere-ADSCs-CM or 3D-ADSCs-CM compared with 2D-ADSCs-CM; the E-cadherin mRNA level was also significantly increased in HepG2 cells after culturing with 3D-ADSCs-CM compared with sphere-ADSCs-CM. In addition, vimentin mRNA expression (in HepG2 cells) was significantly lower after culturing with 3D-ADSCs-CM compared with 2D-ADSCs-CM or sphere-ADSCs-CM. Reduced mRNA expression of N-cadherin (in HepG2 cells) and Zeb1 (in Hcclm3 cells) was observed after culturing with 3D-ADSCs-CM compared with sphere-ADSCs-CM; however, the mRNA expression levels of the other EMT markers were not significantly different among the cells cultured with the various types of ADSCs-CM (Fig. 5). On the basis of these results, it may be suggested that the sphere or 3D culture methods partly enhance the effects of ADSCs on downregulation of the mRNA expression of EMT markers in liver cancer cells. The 3D culture method may be more effective at enhancing the suppressive effects of ADSCs on the mRNA expression levels of EMT markers in liver cancer cells.

To further confirm the effects of ADSCs on translational regulation of EMT, western blotting was used to evaluate the protein expression levels of ETM markers in liver cancer cells. In accordance with the results of RT-qPCR, ADSCs-CM significantly increased E-cadherin protein expression, and decreased the protein expression levels of $\mathrm{N}$-cadherin, vimentin and Zeb1 in liver cancer cells (Fig. 6), which further confirmed the downregulation of EMT in liver cancer cells after culturing with ADSCs-CM.

The results of western blotting indicated that sphere-ADSCs-CM could enhance the downregulation of Zeb1 expression in HepG2 cells compared with 2D-ADSCs-CM; in addition, 3D-ADSCs-CM could further enhance the downregulation of $\mathrm{N}$-cadherin (in Hcclm3 cells), vimentin (in HepG2 cells) and Zeb1 (in Hcclm3 cells) compared with 2D-ADSCs-CM or sphere-ADSCs-CM. However, the expression of other EMT markers was not significantly different among cells cultured with various types of ADSCs-CM (Fig. 6B-E). Therefore, these data confirmed that the sphere or 3D culture methods could partly enhance the effects of ADSCs on downregulation of EMT in liver cancer cells, and the $3 \mathrm{D}$ culture method may be more effective at enhancing the suppressive effects of ADSCs on EMT in liver cancer cells.

\section{Discussion}

Autologous MSCs represent an attractive source for cell-based regenerative medicine, since these cells are present in peripheral blood, bone marrow and nearly all adult tissues or organs, including adipose tissue, dermis, muscle, liver, spleen and lung (39). In particular, ADSCs have been 
A

Heclm3

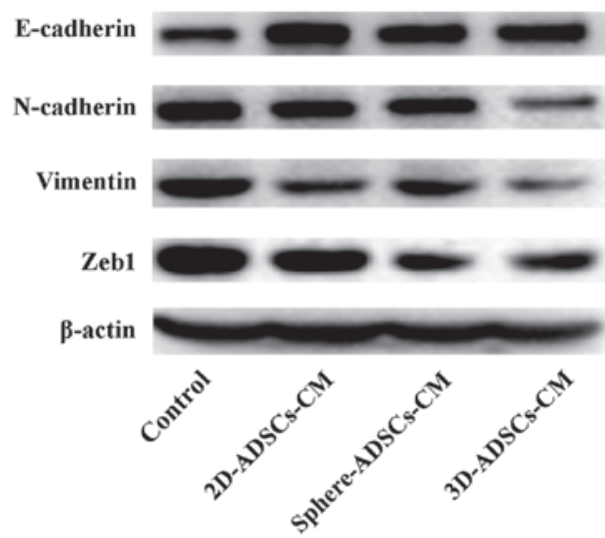

HepG2

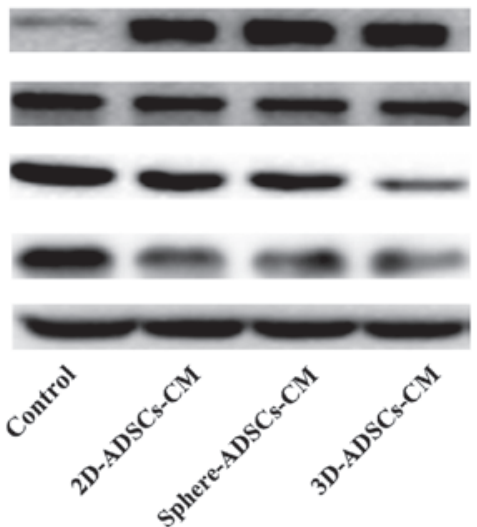

B

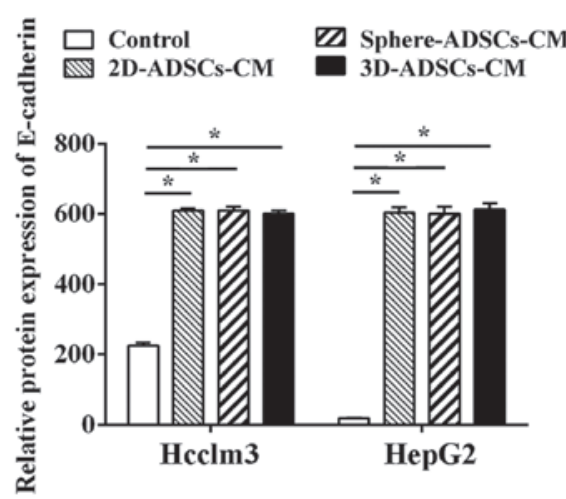

D

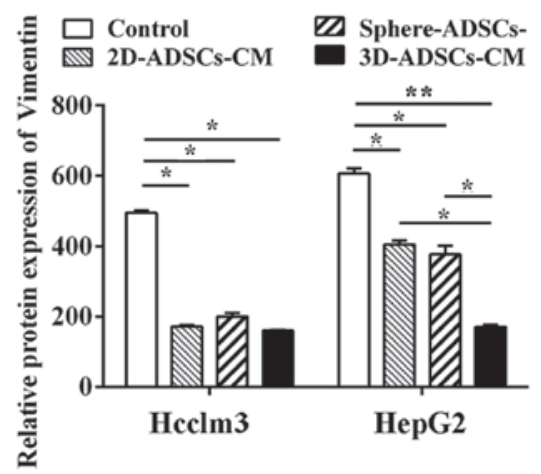

C

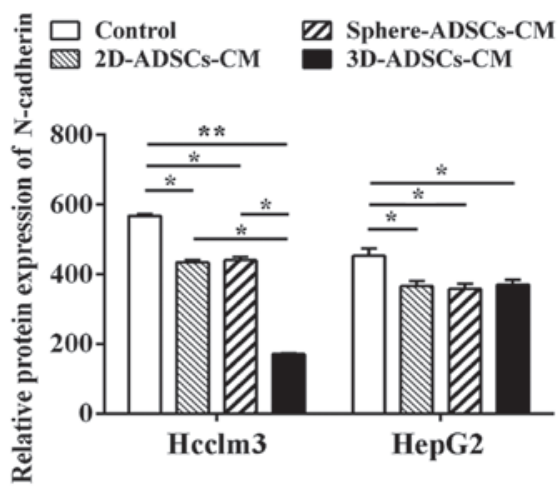

E

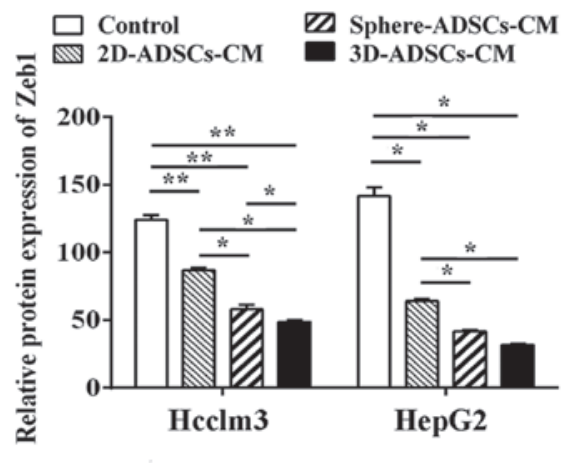

Figure 6. ADSCs-CM downregulates the protein expression levels of EMT markers in liver cancer cells. (A) Western blotting of EMT markers in liver cancer cells after culturing with ADSCs-conditioned medium. Semi-quantitative analysis of (B) E-cadherin, (C) N-cadherin, (D) vimentin and (E) Zeb1 expression in liver cancer cells after culturing with ADSCs-CM. Control, untreated liver cancer cells; 2D-ADSCs-CM, liver cancer cells treated with 2D-ADSCs-CM; sphere-ADSCs-CM, liver cancer cells treated with sphere-ADSCs-CM; 3D-ADSCs-CM, liver cancer cells treated with 3D-ADSCs-CM. For all groups, $\mathrm{n}=3$. $\mathrm{P}<0.05$ and ${ }^{* *} \mathrm{P}<0.01$. 2D, two-dimensional; 3D, three-dimensional; ADSCs-CM, adipose tissue-derived stem cells-conditioned medium; EMT, epithelial-mesenchymal transition; Zebl, zinc finger E-box-binding homeobox 1.

suggested as a novel promising strategy for the treatment of various major diseases, including cardiovascular diseases, liver diseases and cerebral diseases, due to their many advantages, such as increased abundance and easy acquisition compared with other types of MSC $(23-25,40)$. However, the clinical application of ADSCs-based therapy has yet to be implemented, due to numerous aspects. Firstly, the quality control of ADSCs preparation should be confirmed, since various methods of cell isolation and preparation may lead to the transplantation of subtly different cell populations (41). Secondly, the clinical use of ADSCs may be hampered by the lack of understanding of cell behavior following transplantation and the mechanisms by which ADSCs provide therapeutic effects $(41,42)$. Notably, there remain several risks, including adverse events, ectopic tissue formation and in vivo tumorigenic safety after cell transplantation (43). Among these barriers to the clinical application of ADSCs, safety is the prerequisite for ADSCs-based therapy; therefore, the safety of ADSCs has attracted a great deal of interest in cell-based regenerative medicine.

Although it has been clinically proven that autologous ADSCs exhibit short-lived safety for patients $(44,45)$, the 
long-term safety, particularly tumorigenic safety, remains controversial. It has previously been reported that ADSCs may promote tumor growth due to properties of regeneration and vascularization, which are closely associated with tumor initiation and metastasis (46); however, other studies indicated that ADSCs may inhibit tumor progression, due to their attributes, including tumor-homing instinct, immunological characterization, and their capacity for self-renewal and potential for differentiation $(28,29)$. It is generally accepted that molecules secreted from ADSCs may influence the effects of ADSCs on tumor growth. Therefore, the culture conditions of ADSCs may have an important role in determining the association between ADSCs and tumor cells, since various culture conditions could affect the secretion of molecules from ADSCs $(32,33)$. Notably, Tian et al reported that human MSCs may inhibit proliferation of cancer cells in vitro and enhance tumor growth in vivo (34), thus suggesting that MSCs exert a dual effect on the same tumor under various growing conditions. Therefore, the present study used various culture methods, including 2D culture, sphere culture and AlgiMatrix ${ }^{\mathrm{TM}}$ 3D culture, to determine the effects of ADSCs on liver cancer cell growth. The results indicated that ADSCs-CM could inhibit the cell proliferation, motility and adhesive capacity, as well as migration and invasion of liver cancer cells, and could also promote apoptosis of liver cancer cells, thus clearly suggesting that ADSCs may inhibit liver cancer cell growth. It has previously been reported that 2D-ADSCs-CM may inhibit HCC cell (SMMC7721) growth and promote cell death via downregulation of protein kinase B signaling (47). In addition, MSCs have previously effectively inhibited cell growth and promoted apoptosis of HepG2 cells (48). In concordance with these previous results, the present study revealed that ADSCs-CM inhibited cell growth of HCC-derived Hcclm3 cells and hepatoblastoma-derived HepG2 cells.

It has been reported that sphere or $3 \mathrm{D}$ culture methods may promote the secretion of cytokines and chemokines from ADSCs $(32,33)$; consequently, sphere or 3D culture conditions may theoretically enhance the effects of ADSCs on tumor cells. As predicted, the present study demonstrated that sphere or 3D culture methods could enhance the inhibitory effects of ADSCs on liver cancer cell migration, and could accelerate the effects of ADSCs on liver cancer cell apoptosis. Furthermore, more obvious inhibitory effects of ADSCs on liver cancer cell migration could be achieved using the $3 \mathrm{D}$ culture method compared with the sphere culture method. Therefore, the present study indicated that the culture methods could alter the effects of ADSCs on liver cancer cell growth, and the $3 \mathrm{D}$ culture method may be considered the best candidate to enhance the effects of ADSCs on the inhibition of liver cancer cell growth. It has previously been reported that sphere or 3D culture methods could more closely mimic the ADSCs in vivo environment compared with the traditional 2D culture method (49-51). Accordingly, the present study confirmed that ADSCs could inhibit liver cancer cell growth; therefore, ADSCs may exert therapeutic effects in the treatment of patients with liver cancer. However, further in vivo studies are required prior to its clinical use, and further studies regarding the ADSCs-derived antitumor molecules are required, in order to investigate the underlying antitumor mechanisms.
Numerous studies have demonstrated that ADSCs may repair injured tissues by inhibiting inflammation $(24,52)$, and may also inhibit transforming growth factor (TGF)- $\beta 1$ signaling in animal models of experimental peritoneal fibrosis (52). Notably, inflammatory cytokines, such as TGF- $\beta$ and epidermal growth factor, may stimulate EMT of tumor cells (53). Therefore, the present study aimed to determine whether ADSCs could suppress the migration and invasion of liver cancer cells via the inhibition of EMT using RT-qPCR and western blotting. The results indicated that culturing with three types of ADSCs-CM upregulated E-cadherin expression, and downregulated N-cadherin, vimentin and Zeb1 expression, thus suggesting that ADSCs may suppress liver cancer cell migration and invasion via the downregulation of EMT signaling. Therefore, ADSCs may be used to prevent the recurrence and metastasis of liver cancer.

In conclusion, the present study demonstrated that ADSCs may promote liver cancer cell apoptosis, and inhibit liver cancer cell proliferation, motility and adhesion, as well as cell migration and invasion via downregulation of EMT signaling. ADSCs may provide a novel promising therapeutic approach for the treatment of patients with liver cancer. In addition, the 3D culture method could enhance the inhibitory effects of ADSCs on liver cancer cell growth, and may provide a novel approach to explore the association between ADSCs and tumor.

\section{Acknowledgements}

The present study was supported by the specialized Science and Technology Key Project of Fujian Province (grant no. 2013YZ0002-3), the Science and Technology Infrastructure Construction Program of Fujian Province (grant no. 2014Y2005), the Youth Scientific Research Project of Fujian Provincial Health and Family Planning Commission (grant no. 2017-1-85), the Scientific Innovation Project of Fujian Provincial Health and Family Planning Commission (grant no. 2014-CXB-24), the Scientific Foundation of Fuzhou Health Department (grant nos. 2013-S-wq15, 2013-S-wp1, 2014-S-wq-17, 2015-S-wq13 and 2014-S-wq20), and the Project of Fuzhou Science and Technology Department (grant nos. 2014-S-139-3, 2016-s-124-9 and 2016-s-124-4). Parts of this work were presented as an Abstract at the 26th Annual Conference of APASL (February 15-19, 2017, Shanghai, China).

\section{References}

1. Hashemi M and Kalalinia F: Application of encapsulation technology in stem cell therapy. Life Sci 143: 139-146, 2015.

2. Ojeh N, Pastar I, Tomic-Canic M and Stojadinovic O: Stem cells in skin regeneration, wound healing, and their clinical applications. Int J Mol Sci 16: 25476-25501, 2015.

3. Daughtry B and Mitalipov S: Concise review: parthenote stem cells for regenerative medicine: genetic, epigenetic, and developmental features. Stem Cells Transl Med 3: 290-298, 2014.

4. Zhao T, Zhang ZN, Rong Z and Xu Y: Immunogenicity of induced pluripotent stem cells. Nature 474: 212-215, 2011.

5. Panchalingam KM, Jung S, Rosenberg L and Behie LA: Bioprocessing strategies for the large-scale production of human mesenchymal stem cells: a review. Stem Cell Res Ther 6: 225, 2015.

6. Serafini M, Dylla SJ, Oki M, Heremans Y, Tolar J, Jiang Y, Buckley SM, Pelacho B, Burns TC, Frommer S, et al: Hematopoietic reconstitution by multipotent adult progenitor cells: precursors to long-term hematopoietic stem cells. J Exp Med 204: 129-139, 2007. 
7. Al-Nbaheen M, Vishnubalaji R, Ali D, Bouslimi A, Al-Jassir F, Megges M, Prigione A, Adjaye J, Kassem M and Aldahmash A: Human stromal (mesenchymal) stem cells from bone marrow, adipose tissue and skin exhibit differences in molecular phenotype and differentiation potential. Stem Cell Rev 9: 32-43, 2013.

8. Sarugaser R, Lickorish D, Baksh D, Hosseini MM and Davies JE: Human umbilical cord perivascular (HUCPV) cells: a source of mesenchymal progenitors. Stem Cells 23: 220-229, 2005.

9. Prockop DJ: Marrow stromal cells as stem cells for nonhematopoietic tissues. Science 276: 71-74, 1997.

10. Pittenger MF, Mackay AM, Beck SC, Jaiswal RK, Douglas R, Mosca JD, Moorman MA, Simonetti DW, Craig S and Marshak DR: Multilineage potential of adult human mesenchymal stem cells. Science 284: 143-147, 1999.

11. Salem HK and Thiemermann C: Mesenchymal stromal cells: current understanding and clinical status. Stem Cells 28: 585-596, 2010.

12. Cui L, Yin S, Liu W, Li N, Zhang W and Cao Y: Expanded adipose-derived stem cells suppress mixed lymphocyte reaction by secretion of prostaglandin E2. Tissue Eng 13: 1185-1195, 2007.

13. Ivanova-Todorova E, Bochev I, Mourdjeva M, Dimitrov R Bukarev D, Kyurkchiev S, Tivchev P, Altunkova I and Kyurkchiev DS: Adipose tissue-derived mesenchymal stem cells are more potent suppressors of dendritic cells differentiation compared to bone marrow-derived mesenchymal stem cells Immunol Lett 126: 37-42, 2009.

14. Park KJ, Ryoo SB, Kim JS, Kim TI, Baik SH, Kim HJ, Lee KY, Kim M and Kim WH: Allogeneic adipose-derived stem cells for the treatment of perianal fistula in Crohn's disease: a pilot clinical trial. Colorectal Dis 18: 468-476, 2015

15. de la Portilla F, Alba F, García-Olmo D, Herrerías JM, González FX and Galindo A: Expanded allogeneic adipose-derived stem cells (eASCs) for the treatment of complex perianal fistula in Crohn's disease: results from a multicenter phase I/IIa clinical trial. Int J Colorectal Dis 28: 313-323, 2013.

16. Herreros MD, Garcia-Arranz M, Guadalajara H, De-La-Quintana P, Garcia-Olmo D; FATT Collaborative Group: Autologous expanded adipose-derived stem cells for the treatment of complex cryptoglandular perianal fistulas: a phase III randomized clinical trial (FATT 1: fistula Advanced Therapy Trial 1) and long-term evaluation. Dis Colon Rectum 55 762-772, 2012

17. Garcia-Olmo D, Herreros D, Pascual I, Pascual JA, Del-Valle E, Zorrilla J, De-La-Quintana P, Garcia-Arranz M and Pascual M: Expanded adipose-derived stem cells for the treatment of complex perianal fistula: a phase II clinical trial. Dis Colon Rectum 52: 79-86, 2009.

18. García-Olmo D, García-Arranz M, Herreros D, Pascual I, Peiro C and Rodríguez-Montes JA: A phase I clinical trial of the treatment of Crohn's fistula by adipose mesenchymal stem cell transplantation. Dis Colon Rectum 48: 1416-1423, 2005.

19. Mizushima T, Takahashi H, Takeyama H, Naito A, Haraguchi N Uemura M, Nishimura J, Hata T, Takemasa I, Yamamoto $\mathrm{H}$, et al A clinical trial of autologous adipose-derived regenerative cell transplantation for a postoperative enterocutaneous fistula. Surg Today 46: 835-842, 2016

20. Jo CH, Lee YG, Shin WH, Kim H, Chai JW, Jeong EC, Kim JE, Shim H, Shin JS, Shin IS, et al: Intra-articular injection of mesenchymal stem cells for the treatment of osteoarthritis of the knee: a proof-of-concept clinical trial. Stem Cells 32: 1254-1266, 2014

21. Díez-Tejedor E, Gutiérrez-Fernández M, Martínez-Sánchez P, Rodríguez-Frutos B, Ruiz-Ares G, Lara ML and Gimeno BF Reparative therapy for acute ischemic stroke with allogeneic mesenchymal stem cells from adipose tissue: a safety assessment: a phase II randomized, double-blind, placebo-controlled, single-center, pilot clinical trial. J Stroke Cerebrovasc Dis 23: 2694-2700, 2014.

22. Tzouvelekis A, Paspaliaris V, Koliakos G, Ntolios P, Bouros E, Oikonomou A, Zissimopoulos A, Boussios N, Dardzinski B, Gritzalis D, et al: A prospective, non-randomized, no placebo-controlled, phase Ib clinical trial to study the safety of the adipose derived stromal cells-stromal vascular fraction in idiopathic pulmonary fibrosis. J Transl Med 11: 171, 2013

23. Wang B, Ma X, Zhao L, Zhou X, Ma Y, Sun H, Yang Y and Chen B: Injection of basic fibroblast growth factor together with adipose-derived stem cell transplantation: improved cardiac remodeling and function in myocardial infarction. Clin Exp Med 16: 539-550, 2015

24. Seki A, Sakai Y, Komura T, Nasti A, Yoshida K, Higashimoto M, Honda M, Usui S, Takamura M, Takamura T, et al: Adipose tissue-derived stem cells as a regenerative therapy for a mouse steatohepatitis-induced cirrhosis model. Hepatology 58 : $1133-1142,2013$
25. You D, Jang MJ, Kim BH, Song G, Lee C, Suh N, Jeong IG, Ahn TY and Kim CS: Comparative study of autologous stromal vascular fraction and adipose-derived stem cells for erectile function recovery in a rat model of cavernous nerve injury. Stem Cells Transl Med 4: 351-358, 2015.

26. Chu Y, Tang H, Guo Y, Guo J, Huang B, Fang F, Cai J and Wang Z: Adipose-derived mesenchymal stem cells promote cell proliferation and invasion of epithelial ovarian cancer. Exp Cell Res 337: 16-27, 2015.

27. Muehlberg FL, Song YH, Krohn A, Pinilla SP, Droll LH, Leng X, Seidensticker M, Ricke J, Altman AM, Devarajan E, et al: Tissue-resident stem cells promote breast cancer growth and metastasis. Carcinogenesis 30: 589-597, 2009.

28. Yu X, Su B, Ge P, Wang Z, Li S, Huang B, Gong Y and Lin J: Human adipose derived stem cells induced cell apoptosis and $\mathrm{S}$ phase arrest in bladder tumor. Stem Cells Int 2015: 619290, 2015.

29. Sun B, Roh KH, Park JR, Lee SR, Park SB, Jung JW, Kang SK, Lee YS and Kang KS: Therapeutic potential of mesenchymal stromal cells in a mouse breast cancer metastasis model. Cytotherapy 11: 289-298, 2009.

30. Rettinger CL, Fourcaudot AB, Hong SJ, Mustoe TA, Hale RG and Leung KP: In vitro characterization of scaffold-free three-dimensional mesenchymal stem cell aggregates. Cell Tissue Res 358: 395-405, 2014.

31. Ballotta V, Smits AI, Driessen-Mol A, Bouten CV and Baaijens FP: Synergistic protein secretion by mesenchymal stromal cells seeded in 3D scaffolds and circulating leukocytes in physiological flow. Biomaterials 35: 9100-9113, 2014.

32. Bhang SH, Lee S, Shin JY, Lee TJ, Jang HK and Kim BS: Efficacious and clinically relevant conditioned medium of human adipose-derived stem cells for therapeutic angiogenesis. Mol Ther 22: 862-872, 2014

33. Yang CM, Huang YJ and Hsu SH: Enhanced autophagy of adipose-derived stem cells grown on chitosan substrates. Biores Open Access 4: 89-96, 2015.

34. Tian LL, Yue W, Zhu F, Li S and Li W: Human mesenchymal stem cells play a dual role on tumor cell growth in vitro and in vivo. J Cell Physiol 226: 1860-1867, 2011.

35. Pan F, Liao N, Zheng Y, Wang Y, Gao Y, Wang S, Jiang Y and Liu X: Intrahepatic transplantation of adipose-derived stem cells attenuates the progression of non-alcoholic fatty liver disease in rats. Mol Med Rep 12: 3725-3733, 2015.

36. Lopez-Terrada D, Cheung SW, Finegold MJ and Knowles BB Hep G2 is a hepatoblastoma-derived cell line. Hum Pathol 40: $1512-1515,2009$

37. Livak KJ and Schmittgen TD: Analysis of relative gene expression data using real-time quantitative PCR and the 2(-Delta Delta C(T)) Method. Methods 25: 402-408, 2001

38. Voulgari A and Pintzas A: Epithelial-mesenchymal transition in cancer metastasis: mechanisms, markers and strategies to overcome drug resistance in the clinic. Biochim Biophys Acta 1796: 75-90, 2009.

39. Tobita M, Tajima S and Mizuno H: Adipose tissue-derived mesenchymal stem cells and platelet-rich plasma: stem cell transplantation methods that enhance stemness. Stem Cell Res Ther 6: 215, 2015.

40. Bura A, Planat-Benard V, Bourin P, Silvestre JS, Gross F, Grolleau JL, Saint-Lebese B, Peyrafitte JA, Fleury S, Gadelorge M, et al: Phase I trial: the use of autologous cultured adipose-derived stroma/stem cells to treat patients with non-revascularizable critical limb ischemia. Cytotherapy 16: 245-257, 2014.

41. Feisst V, Meidinger S and Locke MB: From bench to bedside: use of human adipose-derived stem cells. Stem Cells Cloning 8: 149-162, 2015.

42. Srijaya TC, Ramasamy TS and Kasim NH: Advancing stem cell therapy from bench to bedside: lessons from drug therapies. J Transl Med 12: 243, 2014.

43. Vériter S, André W, Aouassar N, Poirel HA, Lafosse A, Docquier PL and Dufrane D: Human adipose-derived mesenchymal stem cells in cell therapy: safety and feasibility in different 'Hospital Exemption' clinical applications. PLoS One 10: e0139566, 2015.

44. Fodor PB and Paulseth SG: Adipose derived stromal cell (ADSC) injections for pain management of osteoarthritis in the human knee joint. Aesthet Surg J 36: 229-236, 2016.

45. Pak J, Chang JJ, Lee JH and Lee SH: Safety reporting on implantation of autologous adipose tissue-derived stem cells with platelet-rich plasma into human articular joints. BMC Musculoskelet Disord 14: 337, 2013 
46. Ritter A, Friemel A, Fornoff F, Adjan M, Solbach C, Yuan J and Louwen F: Characterization of adipose-derived stem cells from subcutaneous and visceral adipose tissues and their function in breast cancer cells. Oncotarget 6: 34475-34493, 2015.

47. Zhao W, Ren G, Zhang L, Zhang Z, Liu J, Kuang P, Yin Z and Wang X: Efficacy of mesenchymal stem cells derived from human adipose tissue in inhibition of hepatocellular carcinoma cells in vitro. Cancer Biother Radiopharm 27: 606-613, 2012

48. Hou L, Wang X, Zhou Y, Ma H, Wang Z, He J, Hu H, Guan W and Ma Y: Inhibitory effect and mechanism of mesenchymal stem cells on liver cancer cells. Tumour Biol 35: 1239-1250, 2014.

49. Ylostalo JH, Bartosh TJ, Tiblow A and Prockop DJ: Unique characteristics of human mesenchymal stromal/progenitor cells pre-activated in 3-dimensional cultures under different conditions. Cytotherapy 16: 1486-1500, 2014

50. Cesarz Z and Tamama K: Spheroid culture of mesenchymal stem cells. Stem Cells Int 2016: 9176357, 2016.

51. Khodabandeh Z, Vojdani Z, Talaei-Khozani T, Jaberipour M, Hosseini A and Bahmanpour S: Comparison of the expression of hepatic genes by human Wharton's jelly mesenchymal stem cells cultured in 2D and 3D collagen culture systems. Iran J Med Sci 41: 28-36, 2016.
52. Wakabayashi K, Hamada C, Kanda R, Nakano T, Io $H$, Horikoshi S and Tomino Y: Adipose-derived mesenchymal stem cells transplantation facilitate experimental peritoneal fibrosis repair by suppressing epithelial-mesenchymal transition. J Nephrol 27: 507-514, 2014.

53. Lv N, Gao Y, Guan H, Wu D, Ding S, Teng W and Shan Z: Inflammatory mediators, tumor necrosis factor- $\alpha$ and interferon- $\gamma$, induce EMT in human PTC cell lines. Oncol Lett 10: 2591-2597, 2015

This work is licensed under a Creative Commons Attribution-NonCommercial-NoDerivatives 4.0 International (CC BY-NC-ND 4.0) License. 